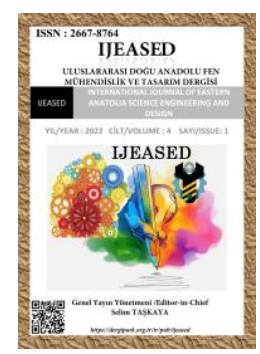

IJEASED

INTERNATIONAL JOURNAL OF EASTERN ANATOLIA

SCIENCE ENGINEERING AND DESIGN

Uluslararası Doğu Anadolu Fen Mühendislik ve Tasarım Dergisi

ISSN: 2667-8764 , 4(1), 1-14, 2022

https://dergipark.org.tr/tr/pub/ijeased

Araştırma Makalesi / Research Article

Doi: $\underline{10.47898 / \text { ijeased.1021497 }}$

\title{
Change of Heating and Cooling Degree-Hours for Different Base Temperatures: A Case Study of Bandırma
}

\author{
Asiye ASLAN *
}

Bandırma Onyedi Eylül University, Department of Electricity and Energy, Gönen, 10900, Turkey.

\begin{tabular}{l|l|l}
\hline \multicolumn{1}{c|}{ Yazar Kimliği / Author ID (ORCID Number) } & \multicolumn{1}{c}{ Makale Süreci / Article Process } \\
\hline "Sorumlu Yazar / Corresponding author : & Geliş Tarihi / Received Date $:$ & 10.11 .2021 \\
aaslan @bandirma.edu.tr & Revizyon Tarihi / Revision Date : & 04.12 .2021 \\
\hline (iD) https://orcid.org/0000-0002-1173-5008 , A. Aslan & Kabul Tarihi / Accepted Date : & 19.12 .2021 \\
& Yayım Tarihi / Published Date : & 15.07 .2022 \\
\hline
\end{tabular}

Alıntı / Cite : Aslan, A. (2022). Change of Heating and Cooling Degree-Hours for Different Base Temperatures: A

Case Study of Bandirma, International Journal of Eastern Anatolia Science Engineering and Design, 4(1), 1-14.

\begin{abstract}
Data on exterior temperature constitute one of the most important parameters in the calculation of energy needs in buildings. This study obtained the temperature distribution curves by analyzing the outdoor air temperature in Bandirma. Heating Degree Hour $(\mathrm{HDH})$ and Cooling Degree Hour $(\mathrm{CDH})$ for three different base temperatures were calculated. A 21-year data set between 2000-2020 obtained from the General Directorate of Meteorology was used. The incidence $(\%)$ of outdoor air temperature according to months was determined. In the calculation of the HDH and CDH values, base temperatures of $\left(15,18\right.$ and $\left.20^{\circ} \mathrm{C}\right)$ and $\left(20,22\right.$ and $\left.24^{\circ} \mathrm{C}\right)$ were considered respectively. The results of the analysis showed that the number of HDH changed between 31357 and 53037, while CDH numbers ranged between 10433 and 2669. The increase in the average outdoor air temperature with the effects of global warming was determined for heating and cooling season in a 21 -year period according to year. It was determined that there was an increase of $1.68{ }^{\circ} \mathrm{C}$ in the heating season in the 21 -year period and $1.80{ }^{\circ} \mathrm{C}$ in the cooling season in the 21 -year period. In this study, the necessity of determining energy needs for heating and cooling purposes in a clear and up-to-date manner was emphasized once again. This is highly important to not only achieve energy savings but also reduce the air pollution effects of energy consumption. It is believed that this study will make a contribution to the literature in this sense.
\end{abstract}

Keywords: Heating degree hours, Cooling degree hours, Energy. 


\section{Farklı Denge Sıcaklıkları İçin Isıtma ve Soğutma Derece-Saatlerinin Değişimi: Bandırma Örneği}

\section{$\ddot{O}_{z e t}$}

Dış sıcaklık verisi binalarda enerji ihtiyacının hesaplanmasında en önemli parametrelerden biridir. Bu çalışmada Bandırma'da dış hava sıcaklık verileri analiz edilerek sıcaklık dağılım eğrileri elde edilmiştir. Üç farklı denge sicaklı̆̆ için Isıtma Derece Saat (IDS) ve Soğutma Derece Saat (SDS) değerleri hesaplanmıştır. Meteoroloji Genel Müdürlüğ̈̈nden temin edilen 2000-2020 yılları arası 21 yıllık veri seti kullanılmıştır. Dış hava sıcaklı̆̆ının aylara göre görülme sıklı̆̆ (\%) belirlenmiştir. IDS ve SDS değerlerinin hesaplanmasında sirasıla (15, 18 ve $\left.20{ }^{\circ} \mathrm{C}\right)$ ve $(20,22$ ve $24^{\circ} \mathrm{C}$ ) denge sıcaklıkları dikkate alınmıştır. Analiz sonuçlarına göre IDS sayısı 31357 ile 53037 arasında değişirken, SDS sayıs ise 10433 ile 2669 arasında değişmektedir. Bununla birlikte 21 yıllık periyotta ısıtma ve soğutma sezonu için küresel ısınma etkileriyle ortalama dış hava sıcaklı̆̆ında yıllara göre artış miktarları belirlenmiştir. Isıtma sezonunda 21 yıllık periyotta $1.68{ }^{\circ} \mathrm{C}$ ve soğutma sezonunda 21 yıllık periyotta $1.80{ }^{\circ} \mathrm{C}$ artış olduğu belirlenmiştir. Bu çalışma ile ısıtma ve soğutma amaçlı enerji ihtiyacının net ve güncel olarak belirlenmesinin gerekliliği bir kez daha vurgulanmıştır. Hem enerji tasarrufu sağlamak hem de enerji tüketiminin hava kirliliği etkisini azalmak için bu oldukça önemlidir. Çalışmanın bu anlamda literatüre katkı sağlaması düşünülmektedir.

Anahtar Kelimeler: Isttma derece-saatler, Soğutma derece-saatler, Enerji.

\section{Introduction}

Energy is one of the most important factors in the development of countries and increasing the wealth level of the society from past to the present. It is extremely important to use energy as efficiently as to obtain it in a clean, reliable, continuous, and economical way.

The prediction of energy needs in buildings is important in terms of calculating the heating and cooling load. Additionally, energy demand is changing constantly with the change in climate conditions. A further increase or decrease in temperature over time also leads to a change in the need for heating and cooling. Although there are many methods used in energy analysis in buildings, one of the simplest and most common of them is the degree-time (degree-day and degreetime) method. The energy need calculated by degree-time values depends on the selected base point temperature. Base point temperature may vary among buildings. Differences can be determined according to the designed indoor temperature and the way of use depending on the thermal properties of the building (Büyükalaca et al., 1999; Şen, 2020).

There are many studies related to the degree-time method in the literature. An et al., 2018 studied the change in heating and cooling day degrees depending on the climate change in Turkey. The temperature degrees obtained by using the RegCM model and calculated heating and cooling day degree values were compared. The comparison was based on the 1981-2000 reference period for the 2016-2035 and 2046-2065 periods. It was stated in the results that there would be a decrease in the heating day degree number and an increase in the cooling day degree numbers across the 
entire country. The study by Altun et al., 2020 examined the impact of outer temperature data on the building heating energy need and the validity of degree day regions formed in standard. The heating energy need of a sample building was calculated according to different outer temperature data of cities and degree day regions in force and the suggested version and compared for 81 provinces. In some between degree day region transitions, it was concluded that the $\mathrm{Q}$ year values of provinces that are in different degree day regions were very close to each other. The study by Dombayc1 and Bayrakçı, 2017 calculated the monthly heating degree-day numbers for 16 provinces in Turkey with the coldest air conditions (Ağrı, Ardahan, Artvin, Bayburt, Bitlis, Erzincan, Erzurum, Gümüşhane, Hakkari, Kars, Kastamonu, Kayseri, Muş, Sivas, Van, and Yozgat). Calculations were repeated for five different control temperatures $\left(\mathrm{Tc}=14,16,18,20\right.$, and $\left.22^{\circ} \mathrm{C}\right)$ The highest degree-day number was obtained in Ardahan, while the lowest degree-day number was obtained in Artvin. The energy consumption for Ardahan was 47\% higher than Artvin in January. The study by Coşkun et al., 2016 examined the temperature changes over $22{ }^{\circ} \mathrm{C}$ for cooling and under $15{ }^{\circ} \mathrm{C}$ for heating by using HadGEM2-ES global climate model. As a result, they stated that the energy consumption would increase with the increase in the need for cooling in summer, while energy consumption would decrease with the decrease in energy need in winter. Pusat et al., 2015 prepared the degree-time (degree-time and degree day) data to use in energy prediction and heat load calculations for Karabük. The obtained measurement data were examined in detail, and missing and problematic parts were separated. They made degree-time calculations and detailed analyses for base temperatures of 5,10 , and $15^{\circ} \mathrm{C}$. The results were presented as tables and compared.

Bandirma is a district of Balikesir province, on the coast of the Marmara Sea. This study obtained the temperature distribution curves and $\mathrm{HDH}$ and $\mathrm{CDH}$ values by analyzing the outdoor temperature data specific to Bandirma. Outdoor dry thermometer temperature data set was used for each month in 21 years, 24 hours a day. Base temperature is usually calculated for the equilibrium temperature of $22{ }^{\circ} \mathrm{C}$ at cooling, $18{ }^{\circ} \mathrm{C}$ at heating for buildings (Bulut et al., 2002; Büyükalaca et al., 2001; Papakostas et al., 2005). According to the comfort diagram drawn by Şen and Kadığlu, it was stated that the comfort temperature range in residences would be between $15{ }^{\circ} \mathrm{C}$ and $24{ }^{\circ} \mathrm{C}$; the need for heating would arise under $15{ }^{\circ} \mathrm{C}$, the need for cooling would arise above $24{ }^{\circ} \mathrm{C}$ (Şen, 1997). Additionally, the cooling threshold value is known to be above $22{ }^{\circ} \mathrm{C}$, and the heating threshold value is known to be under $15^{\circ} \mathrm{C}$, according to HadGEM2-ES global climate model. This study considered all these criteria and both heating $\left(15,18\right.$ and $\left.20^{\circ} \mathrm{C}\right)$ and cooling $(20,22$ and 24 ${ }^{\circ} \mathrm{C}$ ) base temperatures were taken into account. 
In the literature, the annual or monthly degree-day numbers are generally used in the prediction of energy consumption. However, this study aimed to obtain more sensitive predictions by calculating degree-hour values for each month both in the heating and cooling period in terms of contribution to the literature. The results were compared to see the change that may occur based on different base temperatures. The increase in the temperature change based on years was determined by calculating the average temperatures in heating and cooling seasons.

\section{Materials and Methods}

\subsection{Data Analysis}

This study analyzed the outdoor temperature data measured hourly between 2000-2020 for 21 years and obtained from the meteorological station active in Bandirma district. All data were obtained from the Ankara General Directorate of Meteorology. Bandırma station is located at $40^{\circ}$ 19' 52" latitude, $27^{\circ} 59^{\prime} 45^{\prime \prime}$ longitude.

\subsection{Heating and Cooling Degree Hours}

The seasonal energy need for heating and cooling residences and the fuel consumption value associated with this need can be determined based on predetermined architectural designs, the material characteristics of buildings, meteorological temperature measurements and the population of the area. One of the methods for predicting the energy requirement for the heating and cooling of a residence within a period is the degree-time method. Regarding the degree-time method, there are static methods (degree-days, degree-hours etc.), while there are also dynamic methods that are used in calculations made based on the dynamic behavior of the building. In this study, degree-hours is taken into account for energy calculation during heating and cooling buildings. Degree-hours are characterized with the sum of temperature differences between the average outdoor air temperature over a given period of time and a known reference temperature. The number of heating degree hours $(\mathrm{HDH})$ and cooling degree hours $(\mathrm{CDH})$ were determined using equations (1) and (2) (Küçüktopçu and Cemek, 2018; De Rosa et al., 2014; Christenson et al., 2006).

For $T_{\text {out }}<T_{\text {base }}$ 
$H D H=\sum_{1}^{n}\left(T_{\text {base }}-T_{\text {out }}\right)$

For $T_{\text {base }}<T_{\text {out }}$,

$C D H=\sum_{1}^{n}\left(T_{\text {out }}-T_{\text {base }}\right)$

where $\mathrm{n}$ is the days total number during the period. $T_{\text {base }}$ and $T_{\text {out }}$ are base temperature and the mean temperature of outside air, respectively.

\section{Results and Discussion}

This study obtained the temperature distribution curves and $\mathrm{HDH}$ and $\mathrm{CDH}$ values by analyzing the outdoor temperature data specific to Bandırma. Figure 1 shows a 24-hour temperature change by taking a 21 -year average. The highest temperature average in August was $28.37{ }^{\circ} \mathrm{C}$, while the lowest temperature average in January was $3.61{ }^{\circ} \mathrm{C}$. The figure shows that the maximum temperature values were obtained at 15:00 in all months.

In Figure 2, daily change is given on an annual basis by taking the 21-year average. On the 205th-231st days of the years, temperatures above $25{ }^{\circ} \mathrm{C}$ were seen. Table 1 shows the incidence of temperatures. The table shows the distribution $(\%)$ for all months. The highest rate in the heating season was obtained for $4-5{ }^{\circ} \mathrm{C}$, while it was seen at $22-23^{\circ} \mathrm{C}$ in the cooling season.

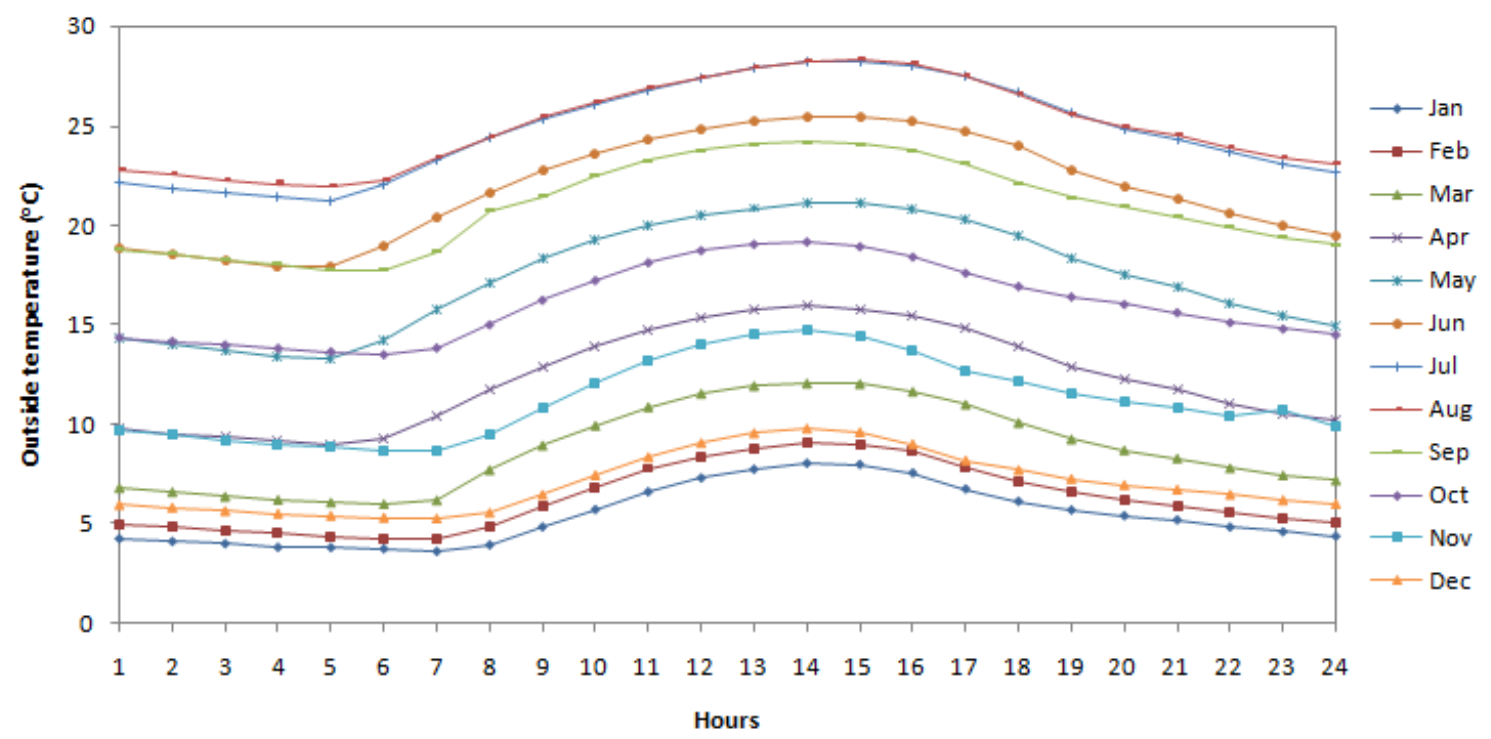

Figure 1. Daily temperature change for each month. 
Aslan, A., International Journal of Eastern Anatolia Science Engineering and Design (IJEASED) / Uluslararası Doğu Anadolu Fen Mühendislik ve Tasarım Dergisi

(2022) 4(1):1-14

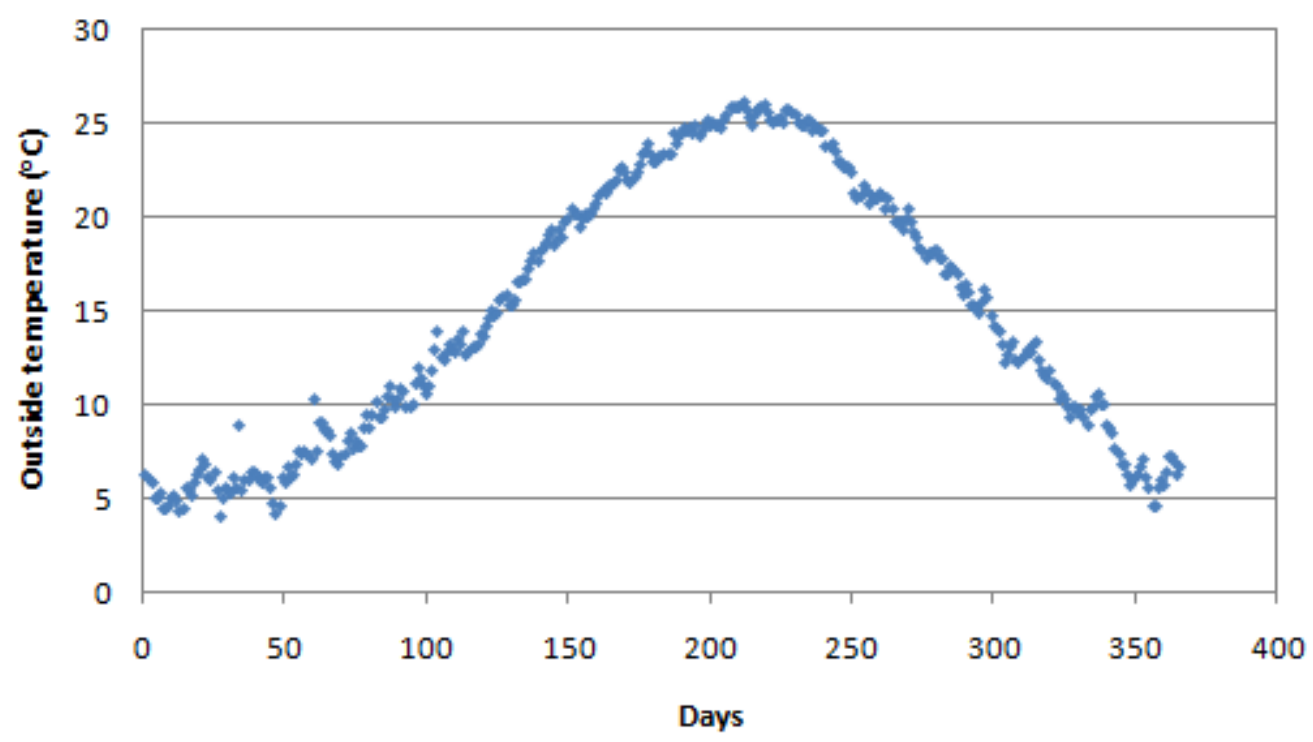

Figure 2. Annual outside temperature distribution.

Table 1. Frequency of temperature values (\%)

\begin{tabular}{ccccccccccccc}
\hline $\begin{array}{c}\text { Temperature } \\
\left({ }^{\mathbf{}} \mathbf{C}\right)\end{array}$ & Jan & Feb & Mar & Apr & May & Jun & Jul & Aus & Sep & Oct & Nov & Dec \\
\hline 0 & 0 & 0 & 0.1 & 0 & 0 & 0 & 0 & 0 & 0 & 0 & 0 & 0 \\
1 & 0.26 & 0.14 & 0 & 0 & 0 & 0 & 0 & 0 & 0 & 0 & 0 & 0 \\
2 & 4.83 & 1.4 & 0 & 0 & 0 & 0 & 0 & 0 & 0 & 0 & 0 & 0.8 \\
3 & 17.7 & 10.3 & 0 & 0 & 0 & 0 & 0 & 0 & 0 & 0 & 0 & 5.7 \\
4 & 23.1 & 20.1 & 2.9 & 0 & 0 & 0 & 0 & 0 & 0 & 0 & 0 & 8.8 \\
5 & 19.6 & 19.7 & 11.8 & 0 & 0 & 0 & 0 & 0 & 0 & 0 & 0 & 18.8 \\
6 & 15.3 & 14.8 & 13.4 & 1.3 & 0 & 0 & 0 & 0 & 0 & 0 & 3 & 19.2 \\
7 & 10.3 & 10.6 & 14.3 & 4.4 & 0 & 0 & 0 & 0 & 0 & 0 & 7.5 & 15.4 \\
8 & 6.1 & 11.5 & 13.8 & 6.3 & 0 & 0 & 0 & 0 & 0 & 0 & 10.8 & 13.5 \\
9 & 1.4 & 8.4 & 12.5 & 9.8 & 0 & 0 & 0 & 0 & 0 & 0.4 & 11.6 & 8.3 \\
10 & 0.9 & 1.2 & 8.7 & 13.3 & 0.5 & 0 & 0 & 0 & 0 & 1.6 & 15.4 & 3.7 \\
11 & 0.1 & 0.4 & 8.7 & 13 & 3.6 & 0 & 0 & 0 & 0 & 3.7 & 15.1 & 2 \\
12 & 0 & 0.1 & 6.8 & 10.1 & 5.9 & 0 & 0 & 0 & 0 & 7.5 & 12 & 1.7 \\
13 & 0 & 0.2 & 5.2 & 9.4 & 8 & 0 & 0 & 0 & 0 & 8.6 & 9.5 & 1.3 \\
14 & 0 & 0.2 & 1.3 & 10.2 & 8.4 & 0 & 0 & 0 & 0.1 & 13.7 & 7.2 & 0.2 \\
15 & 0 & 0.2 & 0 & 8.3 & 10 & 1.2 & 0 & 0 & 0.9 & 15.9 & 4.1 & 0 \\
16 & 0 & 0 & 0 & 2.6 & 9.9 & 3.4 & 0 & 0 & 4 & 14.6 & 2.5 & 0 \\
17 & 0 & 0 & 0 & 0.5 & 10.3 & 4.5 & 0 & 0 & 8.8 & 9 & 0.8 & 0 \\
18 & 0 & 0 & 0 & 0 & 11.2 & 10.8 & 0 & 0 & 14 & 9.1 & 0 & 0 \\
19 & 0 & 0 & 0 & 0 & 6.9 & 11.1 & 0.9 & 0.4 & 12.7 & 7.2 & 0 & 0 \\
20 & 0 & 0 & 0 & 0 & 7.6 & 10.8 & 5.6 & 1.7 & 12.2 & 5.6 & 0 & 0 \\
21 & 0 & 0 & 0 & 0 & 6.8 & 8.4 & 9.8 & 5.3 & 11.6 & 2.6 & 0 & 0 \\
22 & 0 & 0 & 0 & 0 & 6.3 & 11.1 & 12.5 & 16.8 & 10.5 & 0 & 0 & 0 \\
23 & 0 & 0 & 0 & 0 & 3.3 & 12 & 12.2 & 15.3 & 10.9 & 0 & 0 & 0 \\
24 & 0 & 0 & 0 & 0 & 0.5 & 9.8 & 11.2 & 12.3 & 8.3 & 0 & 0 & 0 \\
25 & 0 & 0 & 0 & 0 & 0 & 8.4 & 11.8 & 11.2 & 3.3 & 0 & 0 & 0 \\
26 & 0 & 0 & 0 & 0 & 0 & 6.2 & 11 & 10.3 & 2 & 0 & 0 & 0 \\
27 & 0 & 0 & 0 & 0 & 0 & 1.5 & 11.6 & 13.8 & 0 & 0 & 0 & 0 \\
28 & 0 & 0 & 0 & 0 & 0 & 0.1 & 9.4 & 10 & 0 & 0 & 0 & 0 \\
29 & 0 & 0 & 0 & 0 & 0 & 0 & 3.6 & 2.4 & 0 & 0 & 0 & 0 \\
30 & 0 & 0 & 0 & 0 & 0 & 0 & 0 & 0 & 0 & 0 & 0 & 0 \\
\hline & & & & & & & & & & & \\
\hline
\end{tabular}


Table 2-4 shows the HDH values in the heating season, and Table 5-7 shows the CDH values in the cooling season. The heating seasons consist of (January, February, March, April, October, November, December) months; the cooling season consists of (May, June, July, August, September, October) months. The number of HDH changed between 31357 and 53037, while CDH numbers ranged between 10433 and 2669. When heating and cooling degree hours were compared, it was seen that the heating values were much higher. A $26 \%$ reduction in the number of HDH was observed when the heating base temperature dropped from $18{ }^{\circ} \mathrm{C}$ to $15{ }^{\circ} \mathrm{C}$, and a $24 \%$ increase was observed when it rose from $18{ }^{\circ} \mathrm{C}$ to $20{ }^{\circ} \mathrm{C}$. When the cooling base temperature decreased from $22^{\circ} \mathrm{C}$ to $20^{\circ} \mathrm{C}$, a $78 \%$ increase in the $\mathrm{CDH}$ number was observed, when it increased from $22^{\circ} \mathrm{C}$ to $24^{\circ} \mathrm{C}$, a $55 \%$ decrease was observed. $\mathrm{CDH}$ values changed significantly when the base temperature changed compared to HDH values.

Table 2. HDH at base temperature of $15^{\circ} \mathrm{C}$

\begin{tabular}{ccccccccc}
\hline Time & Jan & Feb & Mar & Apr & Oct & Nov & Dec & Total \\
\hline 00:00-01:00 & 333.1 & 292.9 & 253.5 & 156 & 32.4 & 160.4 & 279.7 & 1508 \\
01:00-02:00 & 337.6 & 296.2 & 260 & 163.9 & 36.1 & 165.9 & 283.9 & 1543.6 \\
02:00-03:00 & 340.9 & 301.9 & 267.2 & 169.3 & 39.6 & 175.6 & 289.3 & 1583.8 \\
03:00-04:00 & 346.7 & 305.1 & 272 & 175 & 42.4 & 179.7 & 293 & 1613.9 \\
04:00-05:00 & 346.7 & 309.2 & 276.4 & 180.6 & 45.4 & 184.9 & 296.5 & 1639.7 \\
05:00-06:00 & 348.9 & 313.5 & 278.9 & 172.6 & 48.6 & 188.9 & 299.2 & 1650.6 \\
06:00-07:00 & 352.9 & 313.3 & 272.8 & 138.2 & 41.8 & 190 & 300.9 & 1609.9 \\
07:00-08:00 & 342.8 & 296.3 & 225.9 & 98 & 22.5 & 164.4 & 291.8 & 1441.7 \\
08:00-09:00 & 314.4 & 265.9 & 187.8 & 62.2 & 10.5 & 125 & 264.2 & 1230 \\
09:00-10:00 & 287.8 & 238.3 & 157.1 & 34.6 & 4.8 & 87.7 & 233.7 & 1044 \\
10:00-11:00 & 259.7 & 120.9 & 128.1 & 20.2 & 1 & 56.1 & 206.6 & 792.6 \\
11:00-12:00 & 237.9 & 191.8 & 107.1 & 11 & 0.3 & 37.2 & 184.8 & 770.1 \\
12:00-13:00 & 224.3 & 180.2 & 95.1 & 7.9 & 0 & 27 & 169.5 & 704 \\
13:00-14:00 & 215 & 170.7 & 91 & 6.8 & 0 & 23 & 162.2 & 668.7 \\
14:00-15:00 & 217 & 173.5 & 92.1 & 6.6 & 0 & 28 & 168 & 685.2 \\
15:00-16:00 & 230.1 & 183.4 & 104.1 & 10.4 & 0.5 & 42.8 & 186.2 & 757.5 \\
16:00-17:00 & 255.3 & 207 & 124.2 & 18.9 & 3.2 & 68.7 & 211.6 & 888.9 \\
17:00-18:00 & 274.6 & 228.7 & 152.5 & 36.7 & 5.3 & 86.3 & 226.2 & 1010.3 \\
18:00-19:00 & 288.2 & 244.8 & 177.8 & 64.7 & 8.3 & 103.1 & 240 & 1126.9 \\
19:00-20:00 & 297 & 264.8 & 195.1 & 82.4 & 11.1 & 115 & 249.4 & 1214.8 \\
20:00-21:00 & 303.8 & 255.9 & 207.8 & 97.8 & 14.9 & 124.9 & 256.1 & 1261.2 \\
21:00-22:00 & 314.9 & 2664.6 & 222.9 & 117.8 & 21.5 & 137.8 & 264 & 3743.5 \\
22:00-23:00 & 322.3 & 273.8 & 234.2 & 133.1 & 25.8 & 147.7 & 272.5 & 1409.4 \\
23:00-24:00 & 328.9 & 282.3 & 241.8 & 144.1 & 30.3 & 154.1 & 278.1 & 1459.6 \\
Total & 7120.8 & 8375 & 4625.4 & 2108.8 & 446.3 & 2774.2 & 5907.4 & 31357.9 \\
\hline
\end{tabular}


Aslan, A., International Journal of Eastern Anatolia Science Engineering and Design (IJEASED) / Uluslararası Doğu Anadolu Fen Mühendislik ve Tasarım Dergisi

(2022) 4(1):1-14

Table 3. $\mathrm{HDH}$ at base temperature of $18^{\circ} \mathrm{C}$

\begin{tabular}{ccccccccc}
\hline Time & Jan & Feb & Mar & Apr & Oct & Nov & Dec & Total \\
\hline 00:00-01:00 & 426.1 & 379.9 & 346.5 & 246 & 113.1 & 250.4 & 372.7 & 2134.7 \\
01:00-02:00 & 430.6 & 383.2 & 353 & 253.9 & 119.7 & 255.9 & 376.9 & 2173.2 \\
02:00-03:00 & 434 & 388.9 & 360.2 & 259.3 & 125.2 & 265.6 & 382.3 & 2215.5 \\
03:00-04:00 & 439.7 & 392.1 & 65 & 265 & 130.4 & 269.7 & 386 & 1947.9 \\
04:00-05:00 & 439.7 & 396.2 & 69.4 & 270.6 & 135.6 & 274.9 & 389.5 & 1975.9 \\
05:00-06:00 & 442 & 400.5 & 371.9 & 262.6 & 140.2 & 278.9 & 392.2 & 2288.3 \\
06:00-07:00 & 445.9 & 400.3 & 365.8 & 228.2 & 129.9 & 280 & 393.9 & 2244 \\
07:00-08:00 & 435.8 & 383.3 & 318.9 & 188 & 93.5 & 254.4 & 384.9 & 2058.8 \\
08:00-09:00 & 407.4 & 352.9 & 380.8 & 152.2 & 57.7 & 215 & 357.2 & 1923.2 \\
09:00-10:00 & 380.8 & 352.3 & 250.1 & 121.2 & 36.9 & 177.7 & 326.7 & 1645.7 \\
10:00-11:00 & 352.7 & 207.9 & 221.1 & 97.2 & 21.1 & 144.6 & 299.6 & 1344.2 \\
11:00-12:00 & 330.8 & 278.8 & 200.1 & 78.3 & 15.2 & 119.3 & 277.8 & 1300.3 \\
12:00-13:00 & 317.3 & 267.2 & 188.1 & 66.9 & 12.6 & 103.2 & 262.5 & 1217.8 \\
13:00-14:00 & 308 & 257.7 & 184 & 61.4 & 12.4 & 97.7 & 255.2 & 1176.4 \\
14:00-15:00 & 310 & 260.5 & 185.1 & 65.9 & 13.8 & 107.3 & 261 & 1203.6 \\
15:00-16:00 & 323.1 & 270.4 & 197.1 & 74.6 & 18.3 & 127.3 & 279.2 & 1290 \\
16:00-17:00 & 348.3 & 294 & 217.2 & 93 & 30.9 & 158.3 & 304.6 & 1446.3 \\
17:00-18:00 & 367.5 & 315.7 & 245.5 & 123.1 & 43.1 & 176.3 & 319.2 & 1590.4 \\
18:00-19:00 & 381.2 & 331.8 & 270.8 & 154.8 & 54.4 & 193.1 & 333 & 1719.1 \\
19:00-20:00 & 390 & 342.9 & 288.1 & 172.5 & 62.8 & 205 & 342.4 & 1803.7 \\
20:00-21:00 & 396.8 & 351.6 & 300.8 & 187.8 & 74.4 & 214.9 & 349.1 & 1875.4 \\
21:00-22:00 & 407.8 & 360.8 & 315.9 & 207.8 & 90 & 227.8 & 357 & 1967.1 \\
22:00-23:00 & 415.2 & 369.3 & 327.2 & 223.1 & 101.1 & 237.7 & 356.5 & 2030.1 \\
23:00-24:00 & 421.9 & 374.9 & 334.8 & 234.1 & 109 & 244.1 & 371.1 & 2089.9 \\
Total & 9352.6 & 8113.1 & 6293 & 4087.5 & 1441.3 & 4879.1 & 8130.5 & 42661.5 \\
\hline
\end{tabular}

Table 4. HDH at base temperature of $20^{\circ} \mathrm{C}$

\begin{tabular}{ccccccccc}
\hline Time & Jan & Feb & Mar & Apr & Oct & Nov & Dec & Total \\
\hline 00:00-01:00 & 488.1 & 437.9 & 408.5 & 306 & 173.1 & 310.4 & 343.7 & 2467.7 \\
01:00-02:00 & 492.5 & 441.2 & 415 & 313.9 & 181.7 & 315.9 & 438.9 & 2599.1 \\
02:00-03:00 & 495.9 & 446.9 & 422.2 & 319.3 & 187.2 & 325.6 & 444.3 & 2641.4 \\
03:00-04:00 & 501.7 & 450.1 & 427 & 325 & 192.4 & 329.7 & 448 & 2673.9 \\
04:00-05:00 & 501.7 & 454.2 & 431.4 & 330.6 & 197.6 & 334.9 & 451.5 & 2701.9 \\
05:00-06:00 & 503.9 & 458.5 & 433.9 & 322.6 & 202.2 & 338.9 & 454.2 & 2714.2 \\
06:00-07:00 & 507.9 & 458.3 & 427.8 & 288.2 & 191.9 & 340 & 455.9 & 2670 \\
07:00-08:00 & 497.8 & 441.3 & 380.9 & 248 & 155.5 & 314.4 & 446.8 & 2484.7 \\
08:00-09:00 & 469.4 & 410.9 & 342.8 & 212.2 & 116.9 & 275 & 419.2 & 2246.4 \\
09:00-10:00 & 442.8 & 383.3 & 312.1 & 181.3 & 86 & 237.7 & 388.7 & 2031.9 \\
10:00-11:00 & 414.7 & 265.9 & 283.1 & 157.2 & 60.4 & 204.6 & 361.6 & 1747.5 \\
11:00-12:00 & 393 & 336.8 & 262.1 & 138.3 & 45.7 & 179.3 & 339.8 & 1695 \\
12:00-13:00 & 379.3 & 325.2 & 250.1 & 126.6 & 39.7 & 163.2 & 324.5 & 1608.6 \\
13:00-14:00 & 370.1 & 315.7 & 246 & 120.6 & 37.5 & 157.3 & 317.2 & 1564.4 \\
14:00-15:00 & 372 & 318.5 & 247.1 & 125.7 & 41.6 & 167.3 & 323 & 1595.2 \\
15:00-16:00 & 385.1 & 328.4 & 259.1 & 134.6 & 54.6 & 187.3 & 341.2 & 1690.3 \\
16:00-17:00 & 410.3 & 352 & 279.2 & 153 & 74.6 & 218.3 & 366.6 & 1854 \\
17:00-18:00 & 429.6 & 373.7 & 307.5 & 183.1 & 95.2 & 236.3 & 381.2 & 2006.6 \\
18:00-19:00 & 443.2 & 389.8 & 332.8 & 214.8 & 111.9 & 253.1 & 395 & 2140.6 \\
19:00-20:00 & 452.1 & 400.9 & 350.1 & 232.5 & 123.6 & 265 & 404.4 & 2228.6 \\
20:00-21:00 & 458.8 & 409.6 & 362.8 & 247.8 & 136.4 & 274.9 & 411.1 & 2301.4 \\
21:00-22:00 & 469.9 & 418.8 & 377.9 & 267.8 & 152 & 287.8 & 419 & 2393.2 \\
22:00-23:00 & 477.3 & 427.3 & 389.2 & 283.1 & 162.1 & 297.7 & 427.5 & 2464.2 \\
23:00-24:00 & 483.9 & 432.9 & 396.8 & 294.1 & 171.3 & 304.1 & 433.1 & 2516.2 \\
Total & 10841 & 9478.1 & 8345.4 & 5526.3 & 2991.1 & 6318.7 & 9536.4 & 53037 \\
\hline & & & & & & & &
\end{tabular}


Table 5. $\mathrm{CDH}$ at base temperature of $20{ }^{\circ} \mathrm{C}$

\begin{tabular}{cccccccc}
\hline Time & May & Jun & Jul & Aug & Sep & Oct & Total \\
\hline 00:00-01:00 & 0 & 3.2 & 67.9 & 88.5 & 2.6 & 0 & 162.2 \\
01:00-02:00 & 0 & 1.3 & 57.9 & 80.4 & 1.1 & 0 & 140.7 \\
02:00-03:00 & 0 & 0 & 50.8 & 71.5 & 0.5 & 0 & 122.8 \\
03:00-04:00 & 0 & 0 & 43.9 & 66.1 & 0 & 0 & 110 \\
04:00-05:00 & 0 & 0 & 38.9 & 61.4 & 0 & 0 & 100.3 \\
05:00-06:00 & 0 & 3.8 & 63.6 & 70 & 0 & 0 & 137.4 \\
06:00-07:00 & 0 & 22.8 & 102.9 & 104.7 & 3.9 & 0 & 234.3 \\
07:00-08:00 & 0 & 50.8 & 138.2 & 139.5 & 19.9 & 0 & 348.4 \\
08:00-09:00 & 5.3 & 81 & 167.2 & 169.6 & 45.9 & 0 & 469 \\
09:00-10:00 & 15.8 & 106.5 & 190.7 & 193 & 74.8 & 0 & 580.8 \\
10:00-11:00 & 27.1 & 127.8 & 212.6 & 213.6 & 97.6 & 2.1 & 680.8 \\
11:00-12:00 & 34.9 & 143 & 230.6 & 232.2 & 114.1 & 7.1 & 761.9 \\
12:00-13:00 & 42.8 & 155 & 245.5 & 246.5 & 123.4 & 10.9 & 824.1 \\
13:00-14:00 & 47.6 & 162.3 & 255.2 & 256.7 & 125.4 & 13 & 860.2 \\
14:00-15:00 & 47.5 & 161.9 & 256.5 & 256.6 & 123.1 & 9.9 & 855.5 \\
15:00-16:00 & 40.2 & 155 & 249.6 & 253 & 112.7 & 5.4 & 815.9 \\
16:00-17:00 & 33.6 & 140.4 & 233.9 & 204 & 92.7 & 0.4 & 705 \\
17:00-18:00 & 18.8 & 118.9 & 209.3 & 204 & 64.3 & 0 & 615.3 \\
18:00-19:00 & 5.4 & 83.6 & 175 & 172 & 45.6 & 0 & 481.6 \\
19:00-20:00 & 1.1 & 57.5 & 149.6 & 153.3 & 34.1 & 0 & 395.6 \\
20:00-21:00 & 0 & 40.8 & 134 & 139.8 & 24.3 & 0 & 338.9 \\
21:00-22:00 & 0 & 24.8 & 114 & 122.5 & 12.8 & 0 & 274.1 \\
22:00-23:00 & 0 & 14.4 & 97.7 & 107.1 & 7.2 & 0 & 226.4 \\
23:00-24:00 & 0 & 7.7 & 84.5 & 96 & 3.9 & 0 & 192.1 \\
Total & 320.1 & 1662.5 & 3570 & 3702 & 1129.9 & 48.8 & 10433.3 \\
\hline & & & & & & &
\end{tabular}

Table 6. $\mathrm{CDH}$ at base temperature of $22{ }^{\circ} \mathrm{C}$

\begin{tabular}{cccccccc}
\hline Time & May & Jun & Jul & Aug & Sep & Oct & Total \\
\hline 00:00-01:00 & 0 & 0 & 15.06 & 28.91 & 0 & 0 & 43.97 \\
01:00-02:00 & 0 & 0 & 10.35 & 22.22 & 0 & 0 & 32.57 \\
02:00-03:00 & 0 & 0 & 7.47 & 15.76 & 0 & 0 & 23.23 \\
03:00-04:00 & 0 & 0 & 5.5 & 11.67 & 0 & 0 & 17.17 \\
04:00-05:00 & 0 & 0 & 3.84 & 9 & 0 & 0 & 12.84 \\
05:00-06:00 & 0 & 0 & 11.12 & 15.49 & 0 & 0 & 26.61 \\
06:00-07:00 & 0 & 0.33 & 40.96 & 43.91 & 0 & 0 & 85.2 \\
07:00-08:00 & 0 & 10.08 & 76.21 & 77.53 & 1.17 & 0 & 164.99 \\
08:00-09:00 & 0 & 27.81 & 105.29 & 107.65 & 9.6 & 0 & 250.35 \\
09:00-10:00 & 0.45 & 47.2 & 128.79 & 131.05 & 24.02 & 0 & 331.51 \\
10:00-11:00 & 2.91 & 67.87 & 150.64 & 151.61 & 41.09 & 0 & 414.12 \\
11:00-12:00 & 6.94 & 83.04 & 168.62 & 170 & 55.59 & 0 & 484.19 \\
12:00-13:00 & 11.04 & 95.06 & 183.55 & 184 & 64.64 & 0 & 538.29 \\
13:00-14:00 & 14.72 & 102.32 & 193.27 & 194.76 & 66.55 & 0 & 571.62 \\
14:00-15:00 & 13.47 & 101.92 & 194.5 & 197.63 & 64.01 & 0 & 571.53 \\
15:00-16:00 & 8.18 & 95.02 & 187.63 & 191.02 & 55 & 0 & 536.85 \\
16:00-17:00 & 5.65 & 80.47 & 171.99 & 171.03 & 39.22 & 0 & 468.36 \\
17:00-18:00 & 1.91 & 59.29 & 147.31 & 142.03 & 19.59 & 0 & 370.13 \\
18:00-19:00 & 0 & 31.19 & 113.07 & 110.02 & 8.71 & 0 & 262.99 \\
19:00-20:00 & 0 & 14.25 & 87.65 & 91.34 & 5.16 & 0 & 198.4 \\
20:00-21:00 & 0 & 5.77 & 72.1 & 77.84 & 2.34 & 0 & 158.05 \\
21:00-22:00 & 0 & 0.74 & 52.11 & 60.57 & 0.34 & 0 & 113.76 \\
22:00-23:00 & 0 & 0 & 37.46 & 45.67 & 0 & 0 & 83.13 \\
23:00-24:00 & 0 & 0 & 26.65 & 36.11 & 0 & 0 & 62.76 \\
Total & 65.31 & 822.44 & 2191.15 & 2288.59 & 467 & 0 & 5834.49 \\
\hline
\end{tabular}


Table 7. $\mathrm{CDH}$ at base temperature of $24{ }^{\circ} \mathrm{C}$

\begin{tabular}{cccccccc}
\hline Time & May & Jun & Jul & Aug & Sep & Oct & Total \\
\hline 00:00-01:00 & 0 & 0 & 0 & 0 & 0 & 0 & 0 \\
01:00-02:00 & 0 & 0 & 0 & 0 & 0 & 0 & 0 \\
02:00-03:00 & 0 & 0 & 0 & 0 & 0 & 0 & 0 \\
03:00-04:00 & 0 & 0 & 0 & 0 & 0 & 0 & 0 \\
04:00-05:00 & 0 & 0 & 0 & 0 & 0 & 0 & 0 \\
05:00-06:00 & 0 & 0 & 0 & 0 & 0 & 0 & 0 \\
06:00-07:00 & 0 & 0 & 2.3 & 0.63 & 0 & 0 & 2.9 \\
07:00-08:00 & 0 & 0 & 18.5 & 17.8 & 0 & 0 & 36.3 \\
08:00-09:00 & 0 & 1.4 & 43.9 & 45.6 & 2.6 & 0 & 93.5 \\
09:00-10:00 & 0 & 7.9 & 66.7 & 69 & 6.2 & 0 & 149.8 \\
10:00-11:00 & 0 & 18.5 & 88.6 & 89.6 & 11.4 & 0 & 208.1 \\
11:00-12:00 & 0 & 28.8 & 106.6 & 108.2 & 16.8 & 0 & 260.4 \\
12:00-13:00 & 0 & 39.4 & 121.5 & 122.5 & 18.9 & 0 & 302.3 \\
13:00-14:00 & 0 & 45.6 & 131.2 & 132.7 & 17.9 & 0 & 327.4 \\
14:00-15:00 & 0 & 45.3 & 132.5 & 135.6 & 13.5 & 0 & 326.9 \\
15:00-16:00 & 0 & 39.6 & 125.6 & 129 & 8 & 0 & 302.2 \\
16:00-17:00 & 0 & 29 & 109.9 & 109.8 & 1.8 & 0 & 250.5 \\
17:00-18:00 & 0 & 15.6 & 85.3 & 80 & 0 & 0 & 180.9 \\
18:00-19:00 & 0 & 2.9 & 51 & 48 & 0 & 0 & 101.9 \\
19:00-20:00 & 0 & 0 & 27.1 & 30.6 & 0 & 0 & 57.7 \\
20:00-21:00 & 0 & 0 & 15.9 & 19.2 & 0 & 0 & 35.1 \\
21:00-22:00 & 0 & 0 & 6.3 & 7.2 & 0 & 0 & 13.5 \\
22:00-23:00 & 0 & 0 & 18 & 1.4 & 0 & 0 & 19.4 \\
23:00-24:00 & 0 & 0 & 0.5 & 0.1 & 0 & 0 & 0.6 \\
Total & 0 & 274 & 1151.4 & 1146.9 & 97.1 & 0 & 2669 \\
\hline
\end{tabular}

Additionally, it was seen that energy consumption would be higher in high base temperature in heating, while it would be less in high base temperature in cooling. Therefore, it is very important to take the necessary actions such as thermal insulation in terms of energy consumption.

Considering the $18{ }^{\circ} \mathrm{C}$ base temperature, the highest monthly $\mathrm{HDH}$ total was observed in January with 9352, and the lowest monthly HDS total was observed in October with 1441 . When considering the $22{ }^{\circ} \mathrm{C}$ base temperature, the highest monthly $\mathrm{CDH}$ total was observed in August with 2288, and the lowest monthly CDH total was observed in October with 0. Looking at hourly values, the highest HDH was observed between the hours of 06:00-07:00 with 445.9, while the highest $\mathrm{CDH}$ was observed between the hours of 14:00-15:00 with 197.63 and the lowest CDH was observed in all hours in October.

Figure 3 shows the $\mathrm{HDH}$ and $\mathrm{CDH}$ values together as a graphic, considering the $18{ }^{\circ} \mathrm{C}$ heating and $22^{\circ} \mathrm{C}$ cooling base temperature. $\mathrm{HDH}$ values were seen to be much higher compared to $\mathrm{CDH}$ values. 


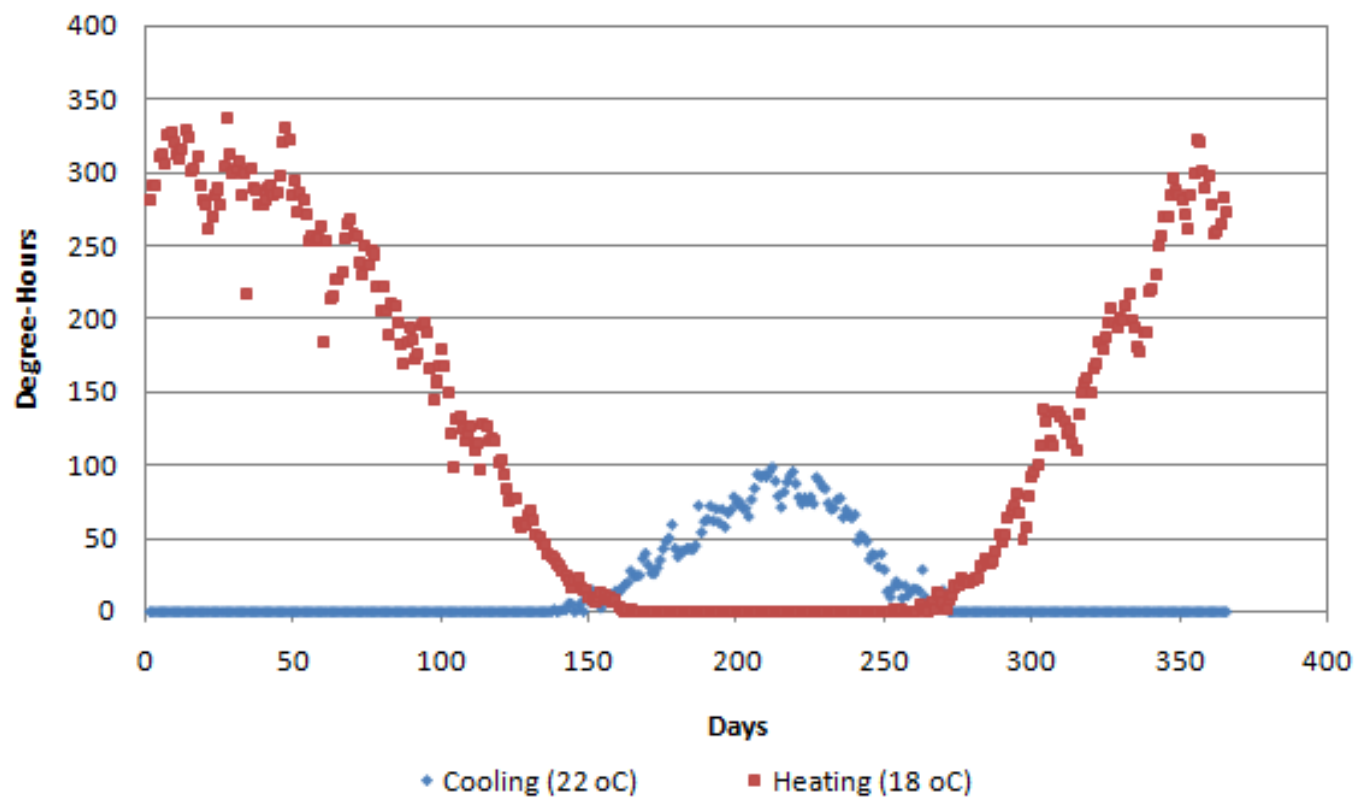

Figure 3. $\mathrm{HDH}$ and $\mathrm{CDH}$ values at $18{ }^{\circ} \mathrm{C}$ and $22{ }^{\circ} \mathrm{C}$ base temperatures.

Figure 4,5 shows temperature changes according to years for the heating and cooling season. Figure 4 shows the average outdoor air temperature of the heating season, and Figure 5 shows the average outdoor air temperature of the cooling season. As can be seen from the figures, there is a trend of increasing temperature values. Between 2000-2020, a temperature increases of $1.68{ }^{\circ} \mathrm{C}$ occurred during the heating season, while a temperature increases of $1.80{ }^{\circ} \mathrm{C}$ occurred during the cooling season. When all months were averaged, an increase of $1.60{ }^{\circ} \mathrm{C}$ occurred. The effects of global warming are clearly visible.

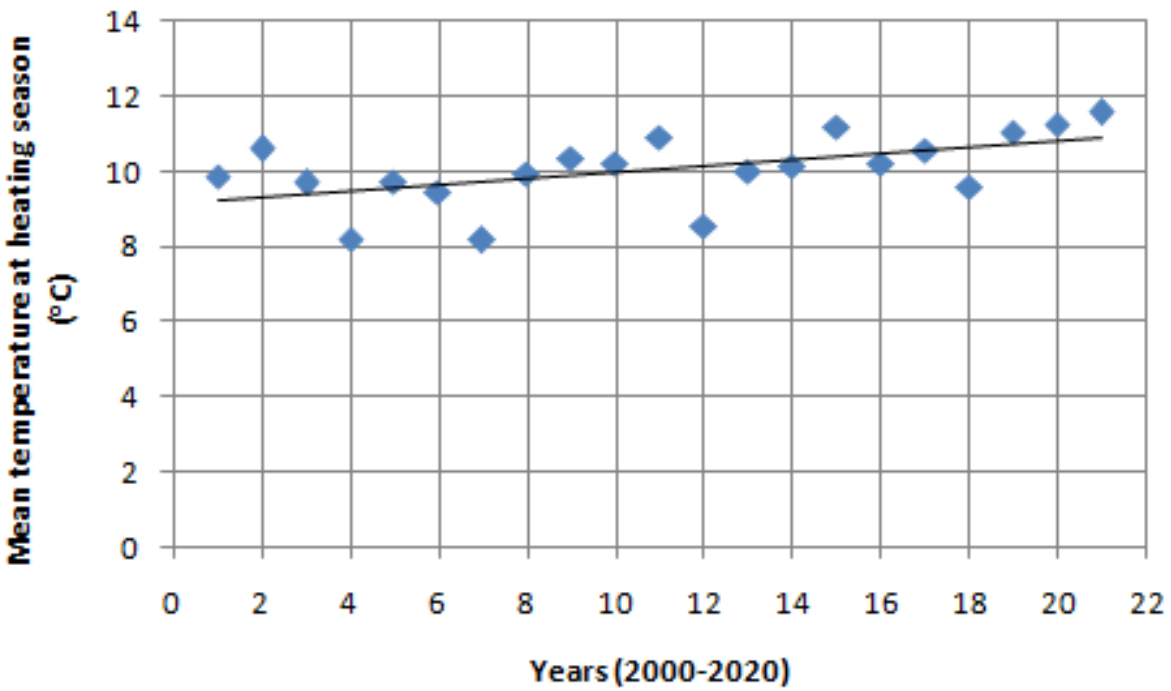

Figure 4. Temperature change over the years in the heating season. 


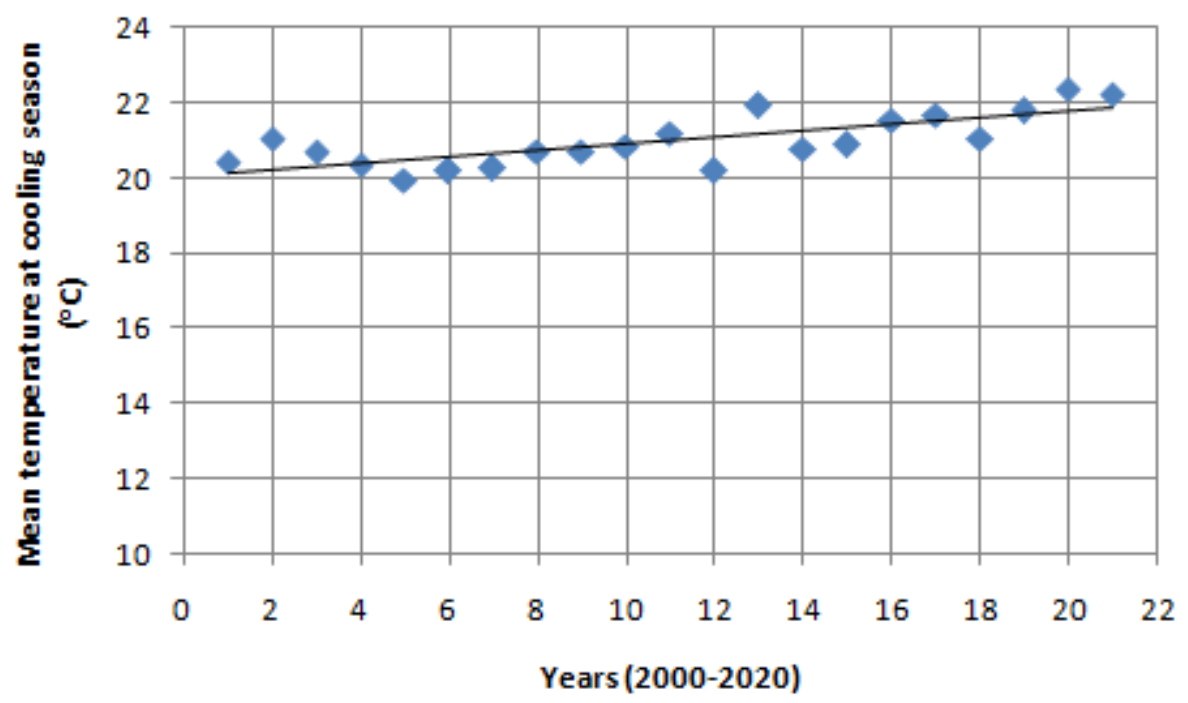

Figure 5. Temperature change over the years in the cooling season.

In this section, the results from this study and the previous literature are compared. In a study conducted for Düzce province in Turkey, when the 32-year heating period was evaluated, it was determined that there was a tendency to increase outdoor air temperature by years, and the average temperature increased from $8.93{ }^{\circ} \mathrm{C}$ to $9.13{ }^{\circ} \mathrm{C}$ with an increase of $0.2{ }^{\circ} \mathrm{C}$ in the 32 -year period (Ertürk et al., 2018). In Bandırma, this increase was achieved as $1.68{ }^{\circ} \mathrm{C}$ in a 21 -year period. The difference in values is more due to the difference in calculated processes than to the regional difference. For Düzce province, a longer process was considered by joining the average of previous years, and for Bandırma, following years were examined compared to Düzce. This reveals that an even greater increase in temperature has occurred from the past to the present. In another study conducted for Sakarya province (Ertürk and Çay, 2021), the CDH value was determined as 10265.1 when the base temperature of $20^{\circ} \mathrm{C}$ was considered, and for Bandirma, this value was 10433 . Considering the base temperature of $24^{\circ} \mathrm{C}$, the $\mathrm{CDH}$ value in Sakarya is 2923.3, and in Bandirma it is 2669. It was observed that there was a greater decrease in CDH value in Bandirma when the base temperature increased. Here, regional differences come forward between provinces. Another study involving overall Turkey stated that in the period of 2016-2035, a decrease in heating day degrees is expected throughout the country and a reverse upward trend is expected in cooling degrees. It is predicted that values may differ in different parts of the country, and that values will be much greater, especially in later years (An et al., 2018). Therefore, it can be stated that similar results were obtained in the previous literature with the results obtained in this study.

As a result, using hourly values both in the heating and cooling energy consumption in buildings give more precise results than annual degree-day numbers. Heating and cooling degree 
values are very different from each other in different months and at different times of the day; therefore, using hourly values will ensure a more sensitive energy consumption prediction. A clear definition of energy consumption; therefore, the use of energy in its most efficient form is very important both in terms of saving and in terms of contributing to the environment.

\section{Conclusion}

This study obtained the temperature distribution curves and HDH and CDH values by analyzing the outdoor temperature data specific to Bandirma. Outdoor dry thermometer temperature data set was used for each month in 21 years, 24 hours a day. Base temperatures were taken into account in heating $\left(15,18\right.$ and $\left.20^{\circ} \mathrm{C}\right)$ and cooling $\left(20,22\right.$ and $\left.24{ }^{\circ} \mathrm{C}\right)$. $\mathrm{CDH}$ values changed significantly when the basic temperature changed compared to HDH values. Additionally, it was determined that there was an upward trend in temperature values between the years 2000-2020; this increase was determined as $1.68{ }^{\circ} \mathrm{C}$ in the heating season and $1.80^{\circ} \mathrm{C}$ in the cooling season. On average, an increase of $1.60{ }^{\circ} \mathrm{C}$ occurred annually. Today, it is necessary to use energy more efficiently or decrease the level of the energy amount used and save energy to eliminate important problems such as global warming. Otherwise, troubled processes may inevitably occur for future generations.

\section{Author Contibution}

I hereby declare that the planning, execution and writing of the article was done by me as the sole author of the article.

\section{Conflict of Interest}

I declare that there is no conflict of interest during the planning, execution and writing of the article.

\section{Statement of Research and Publication Ethic}

In the study, research and publication ethics were complied with.

\section{Acknowledgments}

I would like to thank the Turkish State Meteorological Service for providing the meteorological data from Bandirma meteorological stations. 
Aslan, A., International Journal of Eastern Anatolia Science Engineering and Design (IJEASED) / Uluslararası Doğu Anadolu Fen Mühendislik ve Tasarım Dergisi

(2022) 4(1):1-14

\section{References}

Altun, M., Akçamete, A., Akgül, ÇM. (2020) Investigation of the effect of outdoor temperature data on the building heating energy requirement and validity of the TS 825 degree-day region clustering. Pamukkale University Journal of Engineering Sciences, 26(6), 1062-1075.

An, N., Turp, MT., Akbaş, A., Öztürk, Ö., Kurnaz, ML. (2018) Future Projections of Heating and Cooling Degree Days in a Changing Climate of Turkey. Marmara Journal of Science, 3: 227-240.

Bulut, H., Büyükalaca, O., Yılmaz, T., Aktacir, MA. (2002) GAP bölgesi için detaylı iklim verileri. Harran Üniversitesi GAP IV. Mühendislik Kongresi Bildiriler Kitabl, Şanlıurfa, 183-191.

Büyükalaca, O., Bulut, H., Yılmaz, T. (2001) Analysis of variable-base heating and cooling degree-days for Turkey. Applied Energy, 69(4): 269-283.

Büyükalaca, O., Bulut, H., Yılmaz, T. (1999) Türkiye'nin Bazı İlleri İçin Derece Gün Değerleri. ULIBTK'99-023 12. Ulusal Iss Bilimi ve Tekniği Kongresi, Sakarya, 8-10 Eylül 1999.

Christenson, M., Manz, H., Gyalistras, D. (2006) Climate warming impact on degree-days and building energy demand in Switzerland. Energy Convers Manag 47(6):671-86.

Coşkun, M., Gürkan, H., Arabacı, H., Demircan, M., Eskioğlu, O., Şensoy, S., Yazıcı, B. (2016) İklim Değişikliğinin Enerji Tüketimine Etkisi. 10. Uluslararası Temiz Enerji Sempozyumu (UTES), 24-26 Ekim 2016, İstanbul.

De Rosa, M., Bianco, V., Scarpa, F., Tagliafico, LA. (2014) Heating and cooling building energy demand evaluation; a simplified model and a modified degree days approach. Appl energy128:217-29.

Dombayc1, ÖA., Bayrakçı, HC. (2017) The Calculation Of Monthly Heating Degree-Day Numbers Used To Prediction Of Heating Energy Consumption Of Buildings For In Cold Climate Provinces Of Turkey. SDU Journal Of Technical Sciences, 7;2;18-25.

Ertürk, M., Oktay, Z., Çoşgun, C., Keçebaş, A., Çay, Y., Daşdemir, A. (2018) Investigation of energy and emission change for house heating with context of global warming in Düzce/Turkey. Journal of Polytechnic ISSN: 2147-9429.

Ertürk, M., Çay, Y. (2021) Calculation of building cooling loads using the cooling degree-hour method, Politeknik Dergisi, 24(2): 723-732.

Küçüktopçu, E., Cemek, B. (2018) A study on environmental impact of insulation thickness of poultry building walls. Energy 150, 583-590.

Papakostas, K., Kyriakis, N. (2005) Heating and cooling degree-hours for Athens and Thessaloniki, Greece. Renewable Energy, 30: 1873-1880.

Pusat, Ş., Tunç, N., Ekmekçi, İ., Yetişken, Y. (2015) Karabük İçin Derece-Zaman Hesaplamaları. ISITES2015 Valencia-Spain.

Şen, Y. (2020) Design Methodology Of Insulation Material For Buildings In Duzce Province According To Degree-Time And Temperature Analysis. Düzce Üniversitesi Bilim ve Teknoloji Dergisi, 8 1676-1694.

Şen, Z., Kadioglu, M. (1997) Heating Degree-days for Arid Regions, Energy 23: 1089-1094. 